\title{
Estimativa e incerteza de curvas cota-volume por meio de sensoriamento remoto
}

\section{Estimation and uncertainty of remote-sensing-based Depth-Area-Volume (DAV) relationships}

\author{
Bruno Collischonn ${ }^{1}$ e Robin Thomas Clarke ${ }^{2}$ \\ ${ }^{1}$ Agência Nacional de Águas, Brasília, DF, Brasil \\ ${ }^{2}$ Universidade Federal do Rio Grande do Sul, Porto Alegre, RS, Brasil \\ E-mails: bruno.collischonn@ana.gov.br (BC), clarke@iph.ufrgs.br (RTC)
}

Recebido: Fevereiro 05, 2016 - Revisado: Junho 29, 2016 - Aceito: Junho 30, 2016

\begin{abstract}
RESUMO
Os reservatórios de regularização são os únicos mananciais confiáveis para atendimento à demanda de água em boa parte do semi-árido brasileiro. Além do conhecimento da hidrologia e clima locais, a disponibilidade hídrica destes mananciais depende fundamentalmente da informação sobre a geometria do reservatório, expressa usualmente por meio da curva cota-área-volume. Entretanto, na maior parte dos reservatórios do semi-árido, esta informação se encontra muito desatualizada ou inconsistente, seja por imprecisões nos levantamentos originais ou por assoreamento do manancial. Neste trabalho, propõe-se uma forma barata de cubar reservatórios de regularização, estimando-se sua curva cota-área-volume com base em uma sequência de imagens de satélite e nos dados de nível d'água. Dois métodos para quantificar o intervalo de confiança decorrente dessa estimativa são avaliados. Os resultados foram validados com base em um reservatório que dispõe de batimetria mais atualizada, e a metodologia foi usada para estimar o volume do reservatório do Bico da Pedra (MG), cuja capacidade é objeto de incertezas importantes.
\end{abstract}

Palavras-chave: Curva cota-volume; Sensoriamento remoto; Batimetria.

\begin{abstract}
Artificial reservoirs are the only reliable source of water resources in most of the brazilian semiarid region. Their water availability depends on the hydrology and the climate, but also on the geometry of the reservoir, expressed usually in terms of Depth-Area-Volume (DAV) curves. However, for most public reservoirs in northeastern Brazil, this information is often outdated or inconsistent, because of reservoir silting and the lack of systematic updating and assessment of actual storage capacity. . In this work, we present a quick and low-cost statistical procedure to assess reservoir volume, based on temporal sequences of satellite imagery and water level monitoring. Two methods are evaluated to quantify the confidence intervals from these estimates. The results were validated for a reservoir where a recent and reliable bathymetry is available. The approach was then used to estimate the volume of a reservoir in northern Minas Gerais, which storage capacity is uncertain.
\end{abstract}

Keywords: Depth-volume; Remote sensing; Bathymetry. 


\section{INTRODUÇÃO}

A região semi-árida brasileira é caracterizada, em sua maior parte, por rios intermitentes. Esta característica é função da pouca capacidade de retenção dos solos, de pequena profundidade, oriundos de substratos cristalinos. É função, também, do clima, caracterizado por alta evaporação, baixa precipitação média, alta variabilidade interanual da precipitação e concentração das precipitações em curtos períodos de tempo (TOLEDO; ARAÚJO; ALMEIDA, 2014).

Estes fatores levaram o poder público, desde fins do século XIX, a fomentar políticas para armazenamento de água. Destaca-se, neste contexto, o Departamento Nacional de Obras Contra as Secas (DNOCS), que construiu e opera cerca de 324 reservatórios nos 9 estados do nordeste e no norte de Minas Gerais. Deve-se mencionar também a Companhia de Desenvolvimento dos Vales do São Francisco e Parnaíba (CODEVASF), que opera 19 reservatórios nestas duas bacias, além de diversas secretarias e órgãos estaduais.

A determinação da curva cota-área-volume, para um reservatório existente, é feita convencionalmente por meio de batimetria do manancial, levantando-se as profundidades em vários pontos da área inundada e fazendo a devida integração do volume. No caso do reservatório não encontrar-se cheio no momento da batimetria, o volume adicional acima do nível d'água é estimado por aerofotogrametria.

O custo de realização de uma batimetria depende de vários fatores, primeiramente a localização e acessos ao reservatório, que afetam o custo de mobilização de equipes. Além disso, o volume total do reservatório também influencia, visto que há uma economia de escala à medida em que o volume total aumenta. Reservatórios mais encaixados tendem a apresentar um custo menor por unidade de volume, visto que a área inundada a ser varrida é menor. Por fim, a relação entre o volume armazenado no momento do levantamento e o volume total do reservatório também influencia, visto que em geral é mais barato levantar a área seca do que a área molhada do reservatório. Batimetrias recentes em reservatórios da região semi-árida, contratadas pela Agência Nacional de Águas, tiveram custo entre R $\$ 1200$ e R $\$ 4.400$ por hectômetro cúbico $\left(\mathrm{hm}^{3}\right)$.

Entretanto, são poucos os reservatórios de regularização do semi-árido que têm uma sistemática contínua de estimativa da batimetria. Na maior parte dos casos, o que se dispõe são as curvas cota-área-volume estimadas à época do projeto do açude, muitas delas das décadas de 1950-1970, levantando dúvidas sobre a consistência dessa informação. Por um lado, é de se esperar que tenha ocorrido assoreamento do reservatório desde então, diminuindo sua capacidade de armazenamento. Além disso, o aprimoramento da precisão dos sistemas de posicionamento global e equipamentos de aerofotogrametria e batimetria implica que provavelmente haja incertezas importantes advindas da tecnologia usada na época dos projetos.

Em termos globais, o conhecimento da dinâmica espaço-temporal do armazenamento em lagos e reservatórios é surpreendentemente baixo (ALSDORF; RODRIGUEZ; LETTENMAIER, 2007). Apesar de sua importância para as reservas de água doce, o monitoramento sistemático desses mananciais é muito limitado (GAO, 2015). Mesmo em locais onde existem estações de monitoramento, o acesso a dados sobre o armazenamento é frequentemente vedado ao público em geral, por receio de mau uso de informações estratégicas (DUAN; BASTIAANSSEN, 2013).

Dado o custo expressivo para a atualização das curvas cota-área-volume para o grande número de reservatórios existentes, a sua estimativa por meio de sensoriamento remoto pode ser uma fonte alternativa, rápida e barata de informação (ABILEAH, VIGNUDELLI; SCOZZARI, 2011). Neste trabalho, é feita uma apresentação da metodologia proposta, com uma validação para o reservatório de Epitácio Pessoa (PB), que foi objeto de batimetria recentemente. A mesma metodologia é aplicada para estimar a curva cota-área-volume do reservatório do Bico da Pedra (MG), no qual simulações de balanço hídrico recentes indicam a possibilidade de inconsistências na curva. Duas abordagens são testadas para avaliar os intervalos de confiança advindos das estimativas. Além disso, as limitações do método também são discutidas.

\section{RESERVATÓRIOS E SENSORIAMENTO REMOTO}

O sensoriamento remoto vem sendo crescentemente usado como ferramenta em recursos hídricos, havendo tecnologias atualmente para a estimativa de várias componentes do ciclo hidrológico. É o caso da precipitação (GEBREMICHAEL; HOSSAIN, 2010), da evaporação, por meio do satélite MODIS (TANG et al., 2009), variação do armazenamento na superfície, por meio do satélite GRACE (CASTLE et al., 2014), cobertura de neve e nível d'água em rios e reservatórios.

A área inundada por um lago ou reservatório é facilmente identificada em imagens de satélite com sensores na banda do infravermelho próximo (entre 0,76 e 0,90 micrômetros), em que os corpos d'água absorvem a maior parte da radiação. $O$ índice NDWI (Normalised Difference Water Index), proposto primeiramente por McFeeters (1996), é comumente usado na delimitação de corpos d'água, valendo-se do fato que a água apresenta baixa reflectância tanto no verde $(0,52$ a 0,60 micrômetros) quanto no infravermelho próximo, em contraste com outras superfícies como vegetação e solo.

Uma interessante ferramenta para quantificação do armazenamento de água na porção terrestre do ciclo hidrológico é o satélite GRACE (Gravity Recovery and Climate Experiment), que é capaz de medir flutuações no campo gravitacional de porções da terra e consequentemente de sua massa. Como a maior parte da variação da massa em uma dada porção do território se deve à variação do armazenamento de água, é possível correlacionar os resultados com a variação do volume em aquíferos, no solo e em reservatórios.

Entretanto, a resolução espacial muito baixa deste satélite (1 grau ou cerca de 100km) limita seu uso a aplicações de balanço hídrico em maior escala, e efetivamente impossibilita seu uso para estimativa de volume armazenado em reservatórios com algumas dezenas de $\mathrm{km}^{2}$, como é o caso do presente trabalho.

Mais recentemente, diversos trabalhos têm proposto a estimativa do volume em reservatórios por meio da integração de área e nível d'água estimados por satélite. Neste caso, a área inundada é identificada em imagens de satélite de amplo acesso 
como o LANDSAT, enquanto os níveis d'água são estimados a partir de satélites altimétricos como Envisat, Jason, TopexPoseidon, Altika, ICESAT (PAIVA; DURAND HOSSAIN, 2014). Abileah, Vignudelli e Scozzari (2011) utilizou essa abordagem para estimar os volumes do lago Nasser, no rio Nilo. Já Duan e Bastiaanssen (2013) geraram curvas cota-volume para três reservatórios em diferentes partes do mundo (Lake Mead-EUA, Lago Tana-Etiópia e Lago Ijssel na Holanda), obtendo bons resultados nos dois primeiros. Também Medina et al. (2010) e Sima e Tajrishy (2013) estimaram o armazenamento de reservatórios na Guatemala e no Irã, respectivamente. É de se notar que o maior interesse dessas metodologias se dá justamente em países em desenvolvimento.

Há ainda alguns trabalhos que foram além e buscaram estimar outras variáveis operativas, como a afluência ou defluência do reservatório, como é o caso de Cretaux et al. (2005), no Mar de Aral, o que permitiu traçar projeções quanto às tendências de deplecionamento desse importante manancial. Muala et al. (2014) estimou as defluências de dois reservatórios no rio Nilo, com resultados razoáveis.

Entretanto, os satélites comumente usados nesses trabalhos são dotados de radar altimétrico e foram desenvolvidos prioritariamente para medir níveis d'água sobre o oceano. Este fator, associado a aspectos como a pequena largura da faixa varrida (footprint) e inconsistências devido a desníveis topográficos junto às margens, elimina a possibilidade de monitorar a maior parte dos corpos d'água de médio e pequeno porte com os altímetros atualmente disponíveis (CRETAUX; BIRKETT, 2006; GAO; BIRKETT; LETTENMAIER, 2012).

De fato, o trabalho acima mencionado procurou estimar a relação cota-volume de 34 grandes reservatórios ao redor do globo, sendo 6 no Brasil (UHEs Sobradinho, Itaparica, Três Marias, Tucuruí, Nova Ponte e Ilha Solteira), por meio da integração cota-área, sendo a altimetria estimada por satélites altimétricos e a área inundada por sensores MODIS. A Figura 1 mostra o erro médio relativo, em relação ao volume armazenado, das regressões obtidas, mostrando erro crescente à medida em que a capacidade do reservatório diminui.

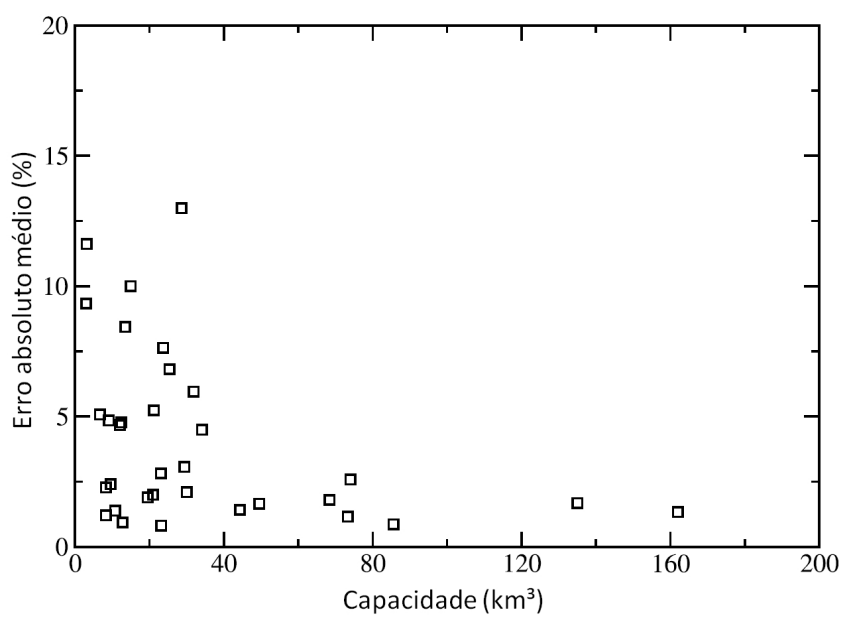

Figura 1. Erro médio em relação ao volume armazenado, obtido da integração de dados de sensoriamento remoto em 34 reservatórios (GAO; BIRKETT; LETTENMAIER, 2012).
Observa-se que a ordem de grandeza dos reservatórios cuja estimativa é possível por esse método é limitada a volumes de algumas dezenas a centenas de quilômetros cúbicos $\left(\mathrm{km}^{3}\right)$, portanto superior à maioria dos reservatórios do semi-árido, que possuem da ordem de dezenas a centenas de hectômetros cúbicos $\left(\mathrm{hm}^{3}\right)$. Em reservatórios de menor porte, o único trabalho correlato é o de Baup, Frappart e Maubant (2014) que estimaram o volume de pequenos reservatórios de irrigação na França de forma completamente remota, com imagens de alta resolução para a delimitação da área inundada e dados de um sensor altimétrico a bordo do satélite ENVISAT.

Embora a precisão ainda seja um fator limitante, estas estimativas possuem a vantagem de dispensar o uso de dados in situ de nível d'água, o que representa uma vantagem em situações em que não há monitoramento do nível d'água ou este não está disponível.

Há certa expectativa na comunidade científica com o lançamento da iniciativa SWOT (Surface Water and Ocean Topography), missão conjunta das agências aeroespaciais da França e dos EUA, que promete superar as limitações relacionadas à altimetria de níveis d'água por sensoriamento. A entrada em operação está prevista para o ano de 2020, e os instrumentos a bordo do satélite serão capazes de monitorar corpos d'água em escalas mais detalhadas, incluindo aí rios mais largos e reservatórios de menor porte, com resolução temporal e espacial mais alta.

No contexto brasileiro e do semi-árido, existem alguns trabalhos correlatos, em que se utilizou de imagens de satélite para inferir informações operacionais de pequenos reservatórios sem monitoramento. É o caso, por exemplo, de Santos et al. (2009), que estimaram volumes de armazenamento na Paraíba, a partir da área inundada estimada em imagens de satélite e em uma relação empírica entre áreas e volumes. Essa abordagem, apoiada em sensoriamento remoto, permite uma avaliação expedita da capacidade de armazenamento em termos de bacia hidrográfica. Cabe destacar também o trabalho de Toledo, Araújo e Almeida, que mapearam a distribuição espacial de pequenos reservatórios no Ceará, chegando a conclusões a respeito de sua vinculação com a geologia, além de gerar um subsídio importante para a simulação de recursos hídricos.

\section{METODOLOGIA}

\section{Formulação geral}

Como mencionado, o presente trabalho se propõe a estimar a curva cota-volume a partir da integração de dados de área inundada e nível d’água, conforme Equação 1:

$V_{i}=\int_{h_{0}}^{h_{i}} A_{i} \cdot d h$

onde Vi é o volume armazenado pelo reservatório em um dado nível $h_{i}$, e $A_{i}$ é área inundada.

Para permitir a integração, a área inundada A é expressa como uma função do nível d'água h, ou seja $\mathrm{A}=\mathrm{f}(\mathrm{h})$, por meio de uma equação de regressão. Foi adotada uma regressão potencial, visto que essa parece ser mais adequada para representar a relação 
entre níveis e áreas inundadas em um reservatório (MOLLE; CADIER, 1992). Assim:

$$
A=a \cdot\left(h-h_{0}\right)^{b}
$$

onde $\mathrm{a}$, b e $\mathrm{h}_{0}$ são parâmetros de ajuste.

Para obtenção de a e b, a Equação 2 é linearizada por meio de transformação logarítmica, ou seja:

$\ln A=\ln a+b \cdot \ln \left(h-h_{0}\right)$

O valor dos parâmetros, bem como sua matriz de covariâncias, são obtido por uma regressão não-linear disponível em Matlab®, que utiliza o algoritmo de mínimos quadrados de Levenberg-Marquardt (SEBER; WILD, 2003).

Para a determinação do volume absoluto, há a necessidade de extrapolação do tramo inferior da relação cota-área, uma vez que dificilmente se dispõe de imagens de satélite em datas em que o reservatório se encontra seco, ou mesmo no volume morto. O parâmetro h0, embora obtido estatisticamente, tem portanto um significado físico, correspondendo aproximadamente ao nível d'água em que a área inundada, e consequentemente o volume, são nulos.

Assim, há um grau de incerteza no tramo inferior da curva cota-área-volume, a qual é minimizada pelo fato de que esse volume em geral tem pouca representatividade na capacidade de armazenamento total.

\section{Estudos de caso}

A metodologia será aplicada a dois reservatórios de regularização na região semi-árida, o reservatório de Epitácio Pessoa ou Boqueirão (PB) e o reservatório do Bico da Pedra (MG). O primeiro foi objeto de uma batimetria recente, realizada em 2013, de forma que será usado para validar a metodologia. Já o segundo possui uma curva cota-volume com inconsistências, de forma que se constitui em uma potencial aplicação da metodologia.

O reservatório de Boqueirão foi construído em 1958 para abastecimento da cidade de Campina Grande, importante polo do interior nordestino, por meio do barramento do rio Paraíba. Gradualmente, sua bacia hidráulica foi ocupada também por usuários de irrigação. Seu volume estimado na época de projeto era superior a $500 \mathrm{hm}^{3}$, entretanto, um levantamento realizado pela SEMARH/PB no ano de 2004 obteve um volume total de $411 \mathrm{hm}^{3}$. A diminuição de $20 \%$ do volume foi atribuída na época ao assoreamento do reservatório. Em 2013, nova batimetria foi realizada pela ANA, somente em cotas inferiores a cerca de $40 \%$ do volume total, resultando em uma curva cota-área volume muito próxima do levantamento anterior, conforme Figura 2.

O reservatório do Bico da Pedra foi construído no fim da década de 1970, barrando o rio Gorutuba, no norte de Minas Gerais. Sua finalidade principal foi atender o perímetro irrigado do Gorutuba, bem como abastecer as cidades de Janaúba e Nova Porteirinha. À época do projeto, o volume armazenado estimado era de $760 \mathrm{hm}^{3}$. Uma nova batimetria realizada em 2007 deu conta de um volume bastante inferior, de $560 \mathrm{hm}^{3}$. Entretanto, relatos do distrito que administra o perímetro sinalizam para a possibilidade do volume ser inferior, visto que o balanço hídrico em períodos sem afluência tem apresentado inconsistências. Por segurança, nos últimos anos o distrito tem tomado decisões sobre a alocação de água considerando um volume $20 \%$ inferior.

\section{Dados de nível d’água}

O nível d'água de ambos os reservatórios é monitorado diariamente. Para o reservatório de Boqueirão, os níveis d'água foram obtidos no sítio web do DNOCS, que opera o açude (DNOCS, 2016).

Já os dados de nível do reservatório do Bico da Pedra são disponibilizados no sitio web do Distrito de Irrigação do Gorutuba (DIG, 2016a), desde 2013. Dados de nível desde a década de 1990 foram obtidos junto à CODEVASF.

A cada imagem de satélite, foi identificado o nível d'água observado no reservatório na data correspondente. No caso do açude Boqueirão, em alguns períodos o monitoramento se deu em frequência semanal ou quinzenal. Nesses casos, o nível correspondente à data da imagem foi estimado por interpolação linear.

O monitoramento de níveis d'água de ambos os açudes tem como referência o zero da régua, não se tratando portanto de níveis absolutos. Este fator não influencia os resultados em termos de volume final, conforme será visto.

\section{Área inundada}

A área inundada pelos reservatórios foi estimada com base em imagens dos seguintes satélites: LANDSAT 5, LANDSAT 7, LANDSAT 8, IRS e CBERS 2. Os dados do LANDSAT 8 foram obtidos de http://earthexplorer.usgs.gov/, enquanto os demais foram obtidos do catálogo de imagens do INPE (DGI, 2016b). A resolução espacial das imagens LANDSAT é de $30 \mathrm{~m}$, enquanto a do IRS é de $24 \mathrm{~m}$ e do CBERS é de $20 \mathrm{~m}$.

Somente imagens sem nuvens sobre a área do reservatório em questão foram selecionadas. Para o reservatório de Boqueirão, um total de 18 imagens entre outubro de 1998 e junho de 2014 foi usado. Já para o reservatório Bico da Pedra, um total de 135 imagens entre fevereiro de 1994 e junho de 2014 foi obtido.

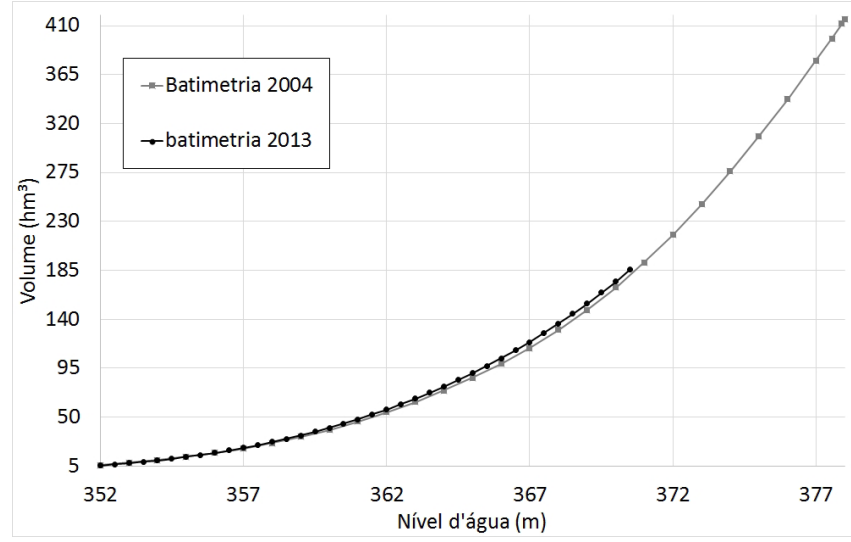

Figura 2. Curvas cota-volume resultantes das batimetrias realizadas em 2004 e 2013, açude Boqueirão. 
Foi desenvolvido um programa em MATLAB para automatização da identificação da área inundada em cada imagem. O programa inicialmente lê o raster correspondente à banda do infravermelho próximo (NIR), e faz um recorte sobre um retângulo envolvente ao reservatório, para evitar o processamento de áreas distantes, sem interesse. Em seguida, é gerado o histograma de reflectâncias da imagem, contando-se o número de pixels em cada intervalo de reflectância. Em imagens de 8 bits (onde a absorção total corresponde a zero e a reflexão total corresponde a 255), as superfícies líquidas têm reflectância geralmente inferior a 50, porém esse valor pode variar em função da nebulosidade, horário do dia e concentração de sedimentos. É possível determinar o limite aproximado identificando-se o ponto onde o histograma muda de declividade, conforme Figura 3.

Aos pixels com valor inferior ao limiar é atribuído um identificador. O programa então delimita as áreas inundadas, por meio da agregação de pixels "úmidos" adjacentes, multiplicando pela área de um pixel.

O programa em MATLAB é capaz de automatizar em cerca de $90 \%$ o trabalho de digitalização de áreas inundadas. No entanto, em alguns casos, a delimitação teve que ser manual, devido à existência de pequenas nuvens contidas sobre a área do reservatório ou excesso de vapor na atmosfera.

\section{Intervalo de confiança - estimativa large sample}

A estimativa do intervalo de confiança para curvas cotavolume oriundas de integração de dados de área e nível d’água não é trivial, visto que a integração acarreta a necessidade de considerar não somente a variabilidade de da área inundada obtida por imagens de satélite, mas também a covariância entre áreas e níveis.

Usando métodos large sample derivados da teoria estatística (ver, por exemplo, STUART; ORD, 1994), o intervalo de confiança do volume, com $95 \%$ de confiabilidade, pode ser expresso por:

$$
I C=V \pm 1,96 \cdot \sqrt{\operatorname{var}[V]}
$$

onde var[V] representa a variância do volume.

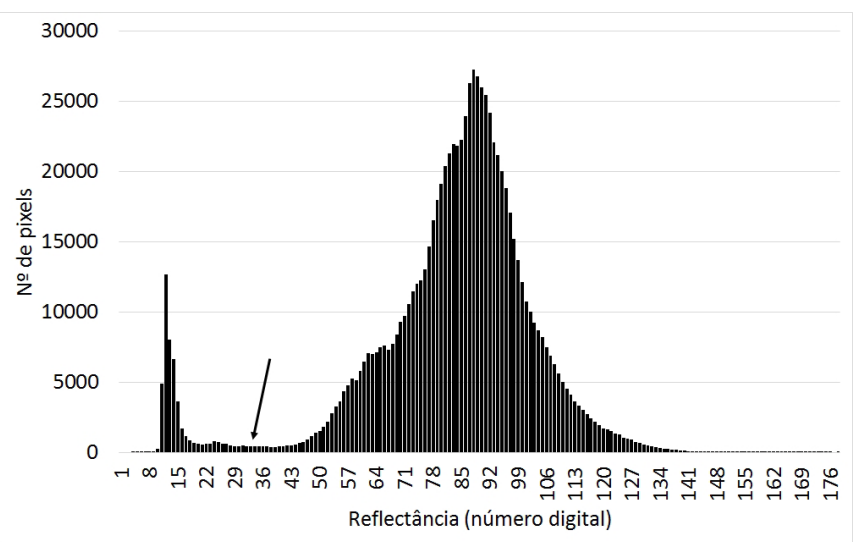

Figura 3. Histograma de reflectância de uma imagem NIR, mostrando o limiar para identificação de áreas inundadas.
Resolvendo numericamente a integral dada pela Equação 1, tem-se:

$V=A_{1} \cdot d h_{1}+A_{2} \cdot d h_{2}+\ldots+A_{n} \cdot d h_{n}$

onde o incremento dh pode ser fixado em um valor próximo da precisão do monitoramento de níveis, $(1 \mathrm{~cm}$ em Boqueirão e $5 \mathrm{~cm}$ em Bico da Pedra).

Com isso, a variância do volume pode ser reescrita conforme abaixo:

$\operatorname{var}[V]=\left(d h_{1}\right)^{2} \cdot \operatorname{var}\left[A_{l}\right]+\left(d h_{2}\right)^{2} \cdot \operatorname{var}\left[A_{2}\right]+\ldots+2 \cdot\left(d h_{1}\right) \cdot\left(d h_{2}\right) \cdot \operatorname{cov}\left[A_{1}, A_{2}\right]+\ldots$

onde $\operatorname{cov}\left[\mathrm{A}_{1}, \mathrm{~A}_{2}\right]$ é a covariância entre as áreas ao longo da curva de regressão.

Em notação matricial, a Equação 6 se reduz a:

$\operatorname{var}[V]=h^{T} W h$

onde $\mathrm{h}^{\mathrm{T}}$ é o vetor $\left[\mathrm{dh}_{1}, \mathrm{dh}_{2}, \ldots \mathrm{dh} \mathrm{h}_{\mathrm{k}}\right]$ transposto, e W é uma matriz com var $\left[A_{1}\right], \operatorname{var}\left[A_{2}\right], \ldots \operatorname{var}\left[A_{k}\right]$ na diagonal principal e cov $\left[A_{i}, A_{j}\right]$ nos elementos de índice $(\mathrm{i}, \mathrm{j})$.

As variâncias e covariâncias de $A_{\text {, }}$, para amostras grandes, são dadas por:

$$
\begin{aligned}
& \operatorname{var}\left[A_{i}\right]=\operatorname{var}\left[a \cdot\left(h_{i}-h_{0}\right)^{b}\right]=\left(\frac{\partial A_{i}}{\partial a}\right)^{2} \cdot \operatorname{var}[a]+ \\
& \left(\frac{\partial A_{i}}{\partial b}\right)^{2} \cdot \operatorname{var}[b]+\left(\frac{\partial A_{i}}{\partial h_{0}}\right)^{2} \cdot \operatorname{var}\left[h_{0}\right]+2 \cdot\left(\frac{\partial A_{i}}{\partial a}\right) \cdot\left(\frac{\partial A_{i}}{\partial b}\right) \\
& \operatorname{cov}[a, b]+2 \cdot\left(\frac{\partial A_{i}}{\partial a}\right) \cdot\left(\frac{\partial A_{i}}{\partial h_{0}}\right) \cdot \operatorname{cov}\left[a, h_{0}\right]+2 \cdot\left(\frac{\partial A_{i}}{\partial b}\right) \\
& \left(\frac{\partial A_{i}}{\partial h_{0}}\right) \cdot \operatorname{cov}\left[b, h_{0}\right]
\end{aligned}
$$

$$
\begin{aligned}
& \operatorname{cov}\left[A_{i}, A_{j}\right]=\frac{\partial A_{i}}{\partial a} \cdot \frac{\partial A_{j}}{\partial a} \cdot \operatorname{var}[a]+\frac{\partial A_{i}}{\partial b} \cdot \frac{\partial A_{j}}{\partial b} \cdot \operatorname{var}[b]+ \\
& \frac{\partial A_{i}}{\partial h_{0}} \cdot \frac{\partial A_{j}}{\partial h_{0}} \cdot \operatorname{var}\left[h_{0}\right]+\left[\frac{\partial A_{i}}{\partial a} \cdot \frac{\partial A_{j}}{\partial b}+\frac{\partial A_{i}}{\partial b} \cdot \frac{\partial A_{j}}{\partial a}\right] \cdot \operatorname{cov}[a, b]+ \\
& {\left[\frac{\partial A_{i}}{\partial a} \cdot \frac{\partial A_{j}}{\partial h_{0}}+\frac{\partial A_{i}}{\partial h_{0}} \cdot \frac{\partial A_{j}}{\partial a}\right] \cdot \operatorname{cov}\left[a, h_{0}\right]+\left[\frac{\partial A_{i}}{\partial b} \cdot \frac{\partial A_{j}}{\partial h_{0}}+\frac{\partial A_{i}}{\partial h_{0}} \cdot \frac{\partial A_{j}}{\partial b}\right] \cdot \operatorname{cov}\left[b, h_{0}\right]}
\end{aligned}
$$

onde:

$$
\begin{aligned}
& \frac{\partial A_{i}}{\partial a}=\left(h_{i}-h_{0}\right)^{b} \\
& \frac{\partial A_{i}}{\partial b}=\left[\ln \left(h_{i}-h_{0}\right)\right] \cdot a \cdot\left(h_{i}-h_{0}\right)^{b} \\
& \frac{\partial A_{i}}{\partial h_{0}}=-a \cdot b \cdot\left(h_{i}-h_{0}\right)^{b-1}
\end{aligned}
$$

Substituindo-se as Equações 10 a 12 nas Equações 8 e 9, tem-se todos os elementos da matriz W em termos das variâncias e covariância de $a$ e $b$, e os intervalos de confiança aproximados (95\%) são

$$
I C=V \pm 1,96 \cdot \sqrt{h^{T} W h}
$$




\section{Intervalo de confiança - estimativa bootstrap}

Este método consiste em integrar a equação de regressão (Equação 2) um grande número de vezes, reamostrando-se os pares A e h, com repetição, a cada iteração.

$\ln A_{r}=a^{\prime}+b^{\prime} \cdot \ln \left(h_{r}-h_{0}^{\prime}\right)$

onde os subíndices $\mathrm{r}$ representam os pares reamostrados, e $a^{\prime}, b^{\prime}$ e $h_{0}$ ' representam os parâmetros da regressão obtidos a partir da amostra (distintos de $a, b$ e $h_{0}$ ).

O número de iterações foi arbitrado em 5000, e a cada nova iteração resulta um volume distinto. Com isso, leva-se em conta a incerteza nos parâmetros $\mathrm{a}, \mathrm{b}$ e $\mathrm{h}_{0}$ da regressão. $\mathrm{O}$ procedimento foi automatizado em MATLAB, e ao fim das 5000 iterações, o programa identifica os percentis do volume com $2,5 \%$ e $97,5 \%$, de forma a estimar os intervalos de confiança com 95\% de confiabilidade.

\section{RESULTADOS}

\section{Reservatório de Boqueirão}

A Figura 4 mostra o resultado da regressão entre áreas inundadas e níveis d'água para o reservatório de Boqueirão.

Entre as imagens dos satélites IRS e CBERS, não foi possível aproveitar nenhuma, devido à constante incidência de nuvens sobre o reservatório.

A hipótese de normalidade dos resíduos, condição para a estimativa "large sample", foi testada por meio do teste de Anderson-Darling, não sendo possível rejeitar a hipótese nula de que os resíduos venham de uma população com distribuição normal, ao nível de significância de 5\%.

Observa-se que houve uma boa amostragem de áreas inundadas próximo ao nível d'água máximo do reservatório $(377,9 \mathrm{~m})$. Por outro lado, imagens em datas em que o reservatório esteve muito baixo, próximo ao volume morto, são naturalmente bastante raras, de forma que há uma incerteza sobre o comportamento do tramo inferior da curva. Essa incerteza, entretanto, tem pouco efeito sobre a curva cota-volume como um todo, uma vez que o

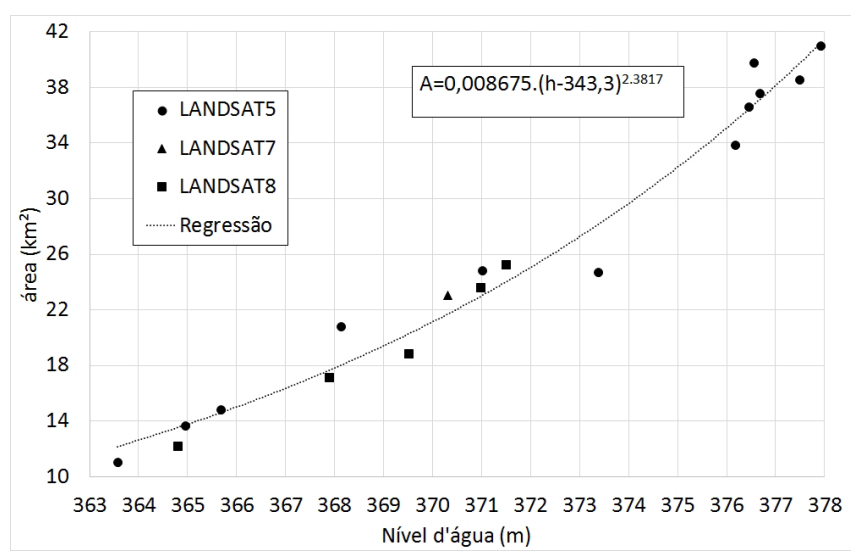

Figura 4. Regressão entre área inundada e nível d'água, açude Boqueirão. tramo não amostrado corresponde a menos de $20 \%$ do volume total do reservatório.

A curva cota-volume resultante da integração e o intervalo de confiança dado pela estimativa "large sample" são mostrados na Figura 5, bem como o volume real do reservatório.

Para a curva do volume real (verdade de campo), foi adotada a curva resultante da batimetria de 2004, visto que a batimetria de 2013 não mostrou grandes diferenças e esteve limitada à porção inferior da curva cota-volume.

$\mathrm{O}$ volume total do reservatório (correspondente à cota $377,9 \mathrm{~m})$, resultante da estimativa por sensoriamento remoto, foi de $411 \mathrm{hm}^{3}, 0,2 \%$ inferior ao volume obtido por batimetria convencional $\left(411,7 \mathrm{hm}^{3}\right)$.

Os limites do intervalo de confiança, para o nível máximo, foram de $353 \mathrm{hm}^{3}$ e $470 \mathrm{hm}^{3}$, portanto o volume máximo real situou-se dentro deste intervalo. Para os demais níveis, a curva cota-volume estimada superestimou levemente a curva de volume real, porém dentro do intervalo de confiança em todas as faixas de nível.

Em termos absolutos, as maiores diferenças entre as curvas cota-volume se dá para níveis d'água em torno de $370 \mathrm{~m}$, de aproximadamente $12 \mathrm{hm}^{3}$. Em termos relativos, o erro é maior para níveis d'água mais baixos, conforme esperado. Para níveis correspondentes ao volume morto, a diferença é de cerca de $10 \%$.

Deve-se levar em conta que a curva de referência, obtida de levantamento batimétrico convencional, também possui incertezas na sua estimativa, decorrentes de erros na medição, amostragem insuficiente de pontos, e interpolação. Assim, é de se esperar que também esta curva possua o seu próprio intervalo de confiança. De fato, a batimetria feita em 2013 no mesmo reservatório, embora restrita a cotas inferiores a $370 \mathrm{~m}$, mostrou volumes um pouco maiores do que a batimetria anterior (Figura 2), portanto mais próximos do que foi obtido pela estimativa de sensoriamento remoto.

A Figura 6 mostra os intervalos de confiança obtidos pela metodologia bootstrap.

Observa-se que o intervalo de confiança gerado por esta metodologia resultou um pouco mais estreito do que a abordagem large sample. Para o nível d'água máximo, o intervalo de confiança do volume ficou entre 398 e $425 \mathrm{hm}^{3}$. Ainda assim, a curva

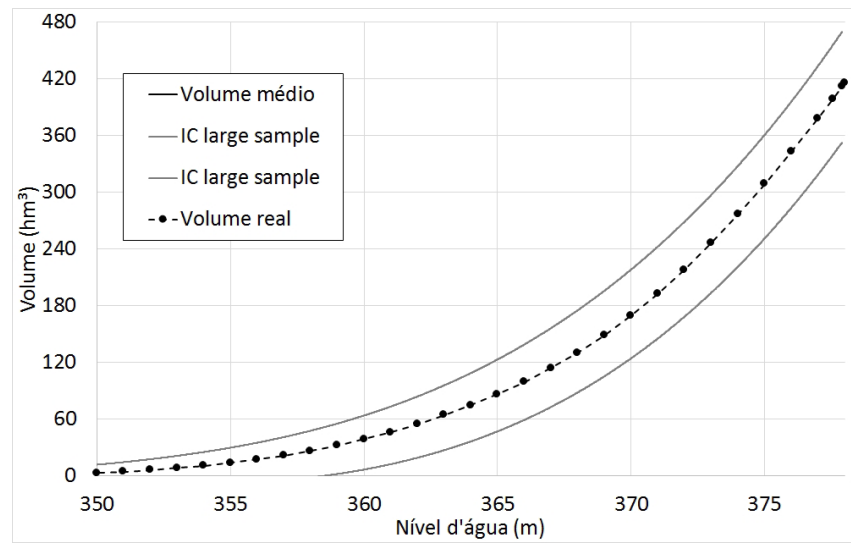

Figura 5. Comparação entre curva cota-volume real do açude Boqueirão e estimada por integração de imagens de satélite, bem como intervalo de confiança pela estimativa large sample. 
cota-volume real manteve-se dentro do intervalo de confiança para todas as faixas de variação.

De forma geral, os resultados podem ser considerados bastante satisfatórios, mesmo com um número relativamente pequeno de imagens disponíveis. As duas metodologias para estimativa do intervalo de confiança levaram a resultados bastante similares. Além disso, a curva aceita como verdade de campo ficou

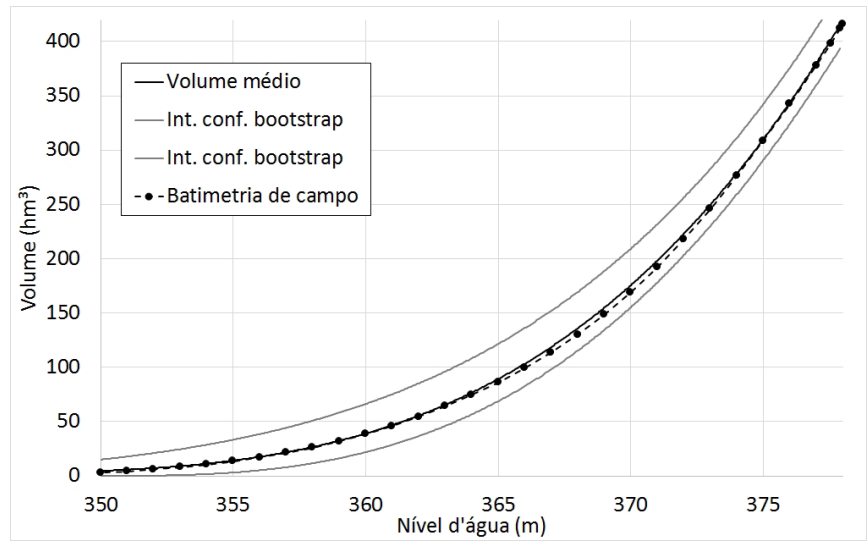

Figura 6. Intervalo de confiança resultante da metodologia bootstrap, açude Boqueirão.

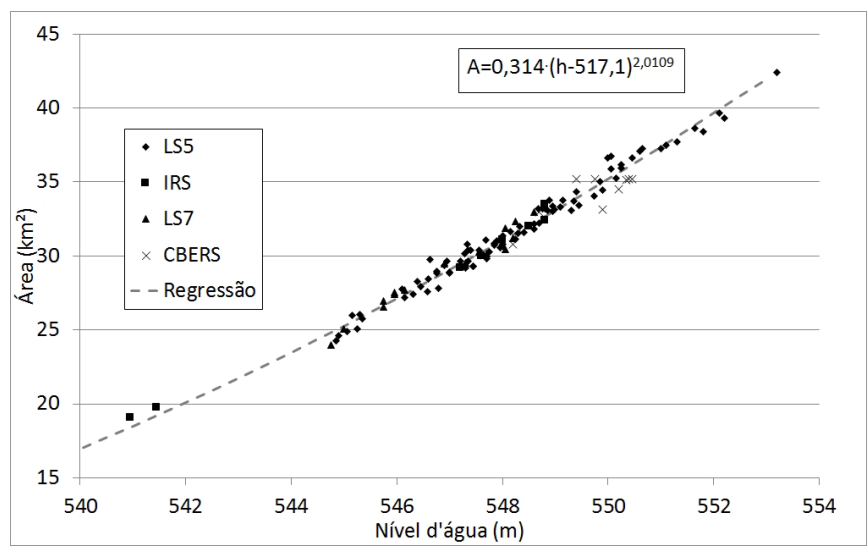

Figura 7. Regressão entre área inundada e nível d'água, açude Bico da Pedra.

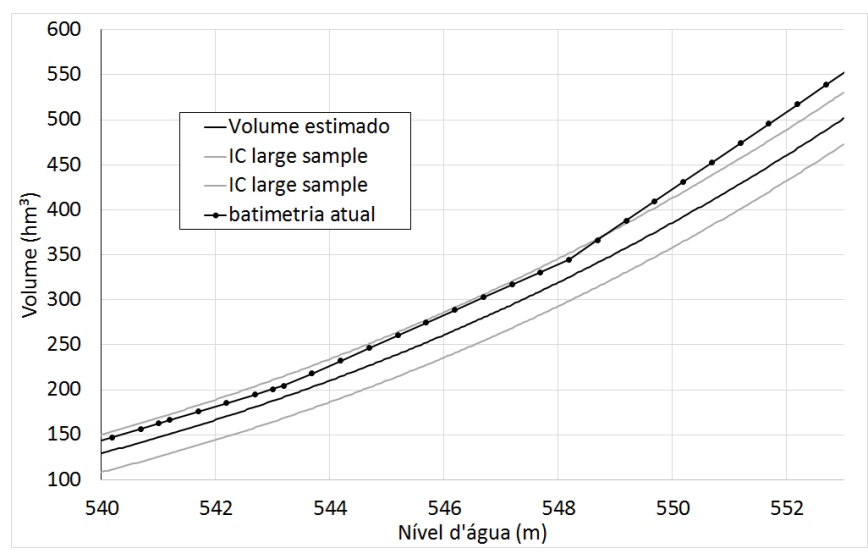

Figura 8. Intervalo de confiança resultante da metodologia large sample, bem como curva cota-volume atualmente usada, açude Bico da Pedra. bastante próxima dos volumes estimados por sensoriamento remoto, sinalizando que a metodologia pode ser uma alternativa interessante para uma avaliação preliminar do volume disponível em reservatórios, quando não se dispõe de batimetrias realizadas em campo.

\section{Reservatório do Bico da Pedra}

Conforme mencionado, no caso deste reservatório a curva cota-volume atualmente disponível tem apresentado inconsistências, não podendo ser adotada como referência (verdade de campo) para validação da metodologia.

A Figura 7 mostra a relação entre área inundada estimada nas imagens de satélite e níveis d'água nas datas correspondentes, bem como a regressão potencial obtida.

Foi possível obter uma boa variação de áreas inundadas nas imagens, inclusive algumas no período de 2013, em que os menores níveis foram observados no reservatório. A porção extrapolada da curva no tramo inferior corresponde a cerca de $25 \%$ do volume total do reservatório. Também nesse caso, o teste de Anderson-Darling não pôde rejeitar a hipótese de normalidade dos resíduos, ao nível de 5\%.

A curva cota-volume resultante da integração e o intervalo de confiança dado pela estimativa "large sample" são mostrados na Figura 8.

Em primeiro lugar, chama a atenção na Figura 8 a discrepância da curva cota-volume resultante da estimativa por sensoriamento remoto, cujo volume máximo (cota 553m) resultou em $502 \mathrm{hm}^{3}$, mais de 10\% inferior ao volume máximo obtido da curva atualmente usada. Este resultado é consistente com relatos do operador do reservatório (DIG, 2013), que tem observado inconsistências no balanço hídrico do reservatório, de forma que tem considerado, por segurança, um volume $20 \%$ inferior ao dado pela curva atualmente usada. Na sua porção superior (cotas acima de $549 \mathrm{~m}$ ) a curva cota-volume "real" situa-se completamente fora do intervalo de confiança.

Assim, entende-se que a curva cota-volume atualmente usada não é um bom parâmetro de comparação para avaliação da metodologia.

Esse resultado é bastante importante pois sinaliza aos gestores do recurso hídrico armazenado no reservatório que o volume disponível é, de fato, inferior ao que era anteriormente estimado.

O intervalo de confiança, para o nível d’água máximo, ficou entre 473 e $530 \mathrm{hm}^{3}$, portanto um intervalo de confiança bem mais estreito do que no caso do açude Boqueirão. Isso se deve principalmente ao número muito maior de imagens disponíveis para estimativa da área inundada, bem como ao melhor ajuste da equação de regressão.

O intervalo de confiança para a curva cota-volume estimado pela metodologia bootstrap resultou muito próximo da estimativa large sample. De fato, julgou-se não ser necessário comparar os intervalos de confiança graficamente, devido à sobreposição dos resultados. Para o nível d'água máximo, o intervalo de confiança ficou entre $473 \mathrm{hm}^{3}$ e $527 \mathrm{hm}^{3}$, um pouco mais estreito do que pela outra metodologia. 
Tabela 1. Sensibilidade do intervalo de confiança à escolha do incremento de nível d'água.

\begin{tabular}{|c|c|c|c|}
\hline $\begin{array}{c}\text { Incremento } \\
\text { (cm) }\end{array}$ & $\begin{array}{c}\text { Limite } \\
\text { inferior } \\
\left(\mathrm{hm}^{3}\right)\end{array}$ & $\begin{array}{c}\text { Limite } \\
\text { superior } \\
\left(\mathrm{hm}^{3}\right)\end{array}$ & $\begin{array}{c}\text { Intervalo } \\
\left(\mathrm{hm}^{3}\right)\end{array}$ \\
\hline 1 & 489 & 515 & 26 \\
\hline 5 & 473 & 530 & 58 \\
\hline 10 & 461 & 542 & 82 \\
\hline 50 & 412 & 592 & 180 \\
\hline
\end{tabular}

Uma constatação adicional diz respeito à escolha do incremento de nível d'água $\Delta \mathrm{h}$ para integração do volume e determinação do intervalo de confiança. Foram testados diferentes incrementos entre 1 e $50 \mathrm{~cm}$, calculando-se os intervalos de confiança para cada um. Embora o volume médio resultante não varie de forma expressiva, os intervalos de confiança estimados pela metodologia large sample foram sensíveis à escolha do valor do incremento, com intervalos de confiança maiores à medida em que o incremento aumenta. A Tabela 1 mostra o resultado dessa análise.

Resultado similar foi obtido para o reservatório do Boqueirão. Já para a estimativa bootstrap, esse resultado não foi observado, uma vez que a largura do intervalo de confiança foi indiferente ao incremento adotado na integração.

Provavelmente a variação do intervalo de confiança com o incremento seja explicada pelo fato de haver uma maior correlação entre os valores da variável independente (ln $\mathrm{A}$ ), à medida em que o valor do incremento diminui (COLLISCHONN; CLARKE, 2016). Esse resultado não invalida a adoção da metologia large sample para estimar a incerteza da curva cota-volume, apenas ressalta o fato de que o incremento a ser usado deve ser escolhido de forma criteriosa, a partir de considerações físicas, como o limite de precisão para medição do nível d’água, o que em geral situa-se entre 1 e $5 \mathrm{~cm}$, no caso do monitoramento dos reservatórios brasileiros.

\section{CONCLUSÕES}

Este artigo apresenta uma metodologia para estimativa da curva cota-área-volume de reservatórios existentes, a partir da integração de imagens de satélite e dados de monitoramento de nível d'água. A metodologia foi validada em um reservatório para o qual se possui um levantamento batimétrico de campo relativamente recente e considerado confiável. Em seguida, foi aplicada em um reservatório sobre o qual há dúvidas quanto à confiabilidade da curva cota-volume atualmente usada, mostrando que de fato esta parece estar superestimada. Duas metodologias foram testadas para o cálculo do intervalo de confiança das curvas cota-volume resultantes, mostrando resultados consistentes entre si.

A metodologia de "batimetria remota" tem algumas limitações para sua aplicação. Em primeiro lugar, só é possível em reservatórios existentes e que operem com regularização, portanto com variação expressiva do nível d'água ao longo do tempo. Assim, não é possível aplicá-la os reservatórios a fio d'água, que constituem boa parte do parque gerador do Sistema Interligado Nacional (SIN).

Devido à resolução das imagens de satélite usadas para delimitar a área inundada, há uma limitação do tamanho do reservatório que poderia ter seu volume estimado pela metodologia apresentada. À medida em que a área inundada diminui, o erro relativo devido à resolução da imagem aumenta. Para as imagens usadas neste trabalho, com resolução de até $30 \mathrm{~m}$, estima-se que somente em reservatórios cuja área inundada mínima seja maior do que $1-2 \mathrm{~km}^{2}$ a metodologia é viável. Isso exclui a maior parte dos reservatórios privados para irrigação existentes no país, que em geral são menores do que esse limiar.

É importante que o intervalo de tempo ao longo do qual as imagens de satélite são obtidas seja relativamente curto, idealmente não mais do que 10 a 15 anos. Isso para evitar que eventuais fenômenos de longo prazo, como assoreamento ou ocupação de margens possam introduzir distorções expressivas na relação cota-área. Naturalmente, nem sempre essa condição é atendida, pela pouca disponibilidade de imagens utilizáveis (sem nuvens) em determinadas regiões.

Nesse sentido, é importante ressaltar ainda que há incertezas não só nos dados obtidos por sensoriamento remoto (área inundada), mas também no dado de monitoramento de nível. A falta de um nivelamento absoluto dos níveis, associada ao eventual deslocamento das réguas, pode significar erros de difícil percepção a longo prazo, o que também reforça a necessidade de limitar o intervalo de tempo para aplicação da metodologia.

Outro pré-requisito é que a metodologia não pode ser aplicada a reservatórios que não contem com histórico de monitoramento de níveis, ou nos quais este não esteja disponível. Isto exclui a maior parte dos reservatórios particulares, bem como reservatórios situados fora do território nacional. Como mencionado, esta limitação está sendo contornada pelo desenvolvimento de novos radares altimétricos a bordo de satélites, para cujo lançamento existe certa expectativa na comunidade acadêmica.

\section{REFERÊNCIAS}

ABILEAH, R.; VIGNUDELLI, S.; SCOZZARI, A. A completely remote sensing approach to monitoring reservoirs water volume. International Water Technology Journal, v. I, n. 1, p. 59-72, 2011.

ALSDORF, D. E.; RODRIGUEZ, E.; LETTTENMAIER, D. P. Measuring surface water from space. Reviews of Geophysics, v. 45, n. 2, p. 1-24, 2007. http://dx.doi.org/10.1029/2006RG000197.

BAUP, F.; FRAPPART, F; MAUBANT, J. Combining highresolution satellite images and altimetry to estimate the volume of small lakes. Hydrology and Earth System Sciences, v. 18, n. 5, p. 2007-2020, 2014. http://dx.doi.org/10.5194/hess-18-2007-2014.

CASTLE, S.; THOMAS, B.; REAGER, J.; RODELL, M.; SWENSON, S.; FAMIGLIETTI, J. S. Groundwater depletion during drought threatens future water security of the Colorado river basin. Geophysical Research Letters, v. 41, n. 16, p. 5904-5911, 2014. PMid:25821273. http://dx.doi.org/10.1002/2014GL061055.

COLLISCHONN, B.; CLARKE, R. Calculating confidence intervals for reservoir volume estimated from remote sensing data. Journal of Hydrologic Engineering-ASCE, 2016. No prelo. 
CRETAUX, J. F.; BIRKETT, C. Lake studies from satellite radar altimetry. Comptes Rendus Geoscience, v. 338, n. 14-15, p. 1098-1112, 2006. http://dx.doi.org/10.1016/j.crte.2006.08.002.

CRETAUX, J. F.; KOURAEV, A. V.; PAPA, F.; BERGÉ-NGUYEN, M.; CAZENAVE, A.; ALADIN, N.; PLOTNIKOV, I. S. Evolution of sea level of the Big Aral Sea from satellite altimetry and its implications for water balance. Journal of Great Lakes Research, v. 31, n. 4, p. 520-534, 2005. http://dx.doi.org/10.1016/S03801330(05)70281-1.

DIG - DISTRITO DE IRRIGAÇÃO DO GORUTUBA. Comunicação pessoal do gerente. Nova Porteirinha, 2013.

DIG - DISTRITO DE IRRIGAÇÃO DO GORUTUBA. Nova Porteirinha, 2016a. Disponível em: <http://www.dig.org.br/ home/>. Acesso em: 5 fev. 2016.

DIG - DISTRITO DE IRRIGAÇÃO DO GORUTUBA. Catálogo de imagens. Nova Porteirinha, 2016b. Disponível em: <http://www. dgi.inpe.br/CDSR/>. Acesso em: 5 fev. 2016.

DNOCS - DEPARTAMENTO NACIONAL DE OBRAS CONTRA AS SECAS. Monitoramento de reservatórios. Fortaleza, 2016. Disponível em: < http://www.dnocs.gov.br/ dnocs/php/ comunicacao/monitoramento_de_reservatorios.php $>$. Acesso em: 5 fev. 2016.

DUAN, Z.; BASTIAANSSEN, W. G. M. Estimating water volume variations in lakes and reservoirs from four operational satellite altimetry databases and satellite imagery data. Remote Sensing of Environment, v. 134, p. 403-416, 2013. http://dx.doi.org/10.1016/j. rse.2013.03.010.

GAO, H. Satellite remote sensing of large lakes and reservoirs: from elevation and area to storage. WIREs Water, v. 2, n. 2, p. 147-157, 2015. http://dx.doi.org/10.1002/wat2.1065.

GAO, H.; BIRKETTT, C.; LETTENMAIER, D. P. Global monitoring of large reservoir storage from satellite remote sensing. Water Resources Research, v. 48, n. W09504, 2012.

GEBREMICHAEL, M.; HOSSAIN, F. Satellite rainfall applications for surface bydrology. Netherlands: Springer, 2010. 327 p. http:// dx.doi.org/10.1007/978-90-481-2915-7.

MCFEETERS, S. K. The use of the Normalized Difference Water Index (NDWI) in the delineation of open water features. International Journal of Remote Sensing, v. 17, n. 7, p. 1425-1432, 1996. http://dx.doi.org/10.1080/01431169608948714.

MEDINA, C.; GOMEZ-ENRI, J.; ALONSO, J. J.; VILLARES, P. Water volume variations in Lake Izabal (Guatemala) from in situ measurements and ENVISAT Radar Altimeter (RA-2) and Advanced Synthetic Aperture Radar (ASAR) data products. Journal of Hydrology (Amsterdam), v. 382, n. 1-4, p. 34-48, 2010. http:// dx.doi.org/10.1016/j.jhydrol.2009.12.016.

MOLLE, F.; CADIER, E. Manual do pequeno açude. Recife: SUDENEDPG-PRN,DPP-WR, 1992. 525 p. Anexos.

MUALA, E.; MOHAMED, Y. A.; DUAN, Z.; VAN DER ZAAG, P. Estimation of reservoir discharges from lake Nasser and Roseires reservoir in the Nile basin using satellite altimetry and imagery data. Remote Sensing, v. 6, n. 8, p. 7522-7545, 2014. http://dx.doi. org/10.3390/rs6087522.

PAIVA, R.; DURAND, M.; HOSSAIN, F. Spatiotemporal interpolation of discharge across a river network by using sinthetic SWOT satellite data. Water Resources Research, v. 51, n. 1, p. 430-449, 2014. http://dx.doi.org/10.1002/2014WR015618.

SANTOS, F.A.; SILANS, A.; PORTO, R.; ALMEIDA, C. Estimativa e análise do volume dos pequenos açudes através de imagem de satélite e levantamento de campo na bacia hidrográfica do Açude Sumé. In: SIMPÓSIO BRASILEIRO DE RECURSOS HÍDRICOS, 18., 2009, Campo Grande. Anais... Porto Alegre: ABRH, 2009.

SEBER, G.; WILD, C. Nonlinear regression. Hoboken: WileyInterscience, 2003.

SIMA, S.; TAJRISHY, M. Using satellite data to extract volumearea-elevation relationships for Urmia Lake, Iran. Journal of Great Lakes Research, v. 39, n. 1, p. 90-99, 2013. http://dx.doi. org/10.1016/j.jglr.2012.12.013.

STUART, A.; ORD, K. Kendall's advanced theory of statistics: distribution theory. 6. ed. Oxford: University Press, 1994. v. 1.

TANG, Q.; PETERSON, S.; CUENCA, R.; HAGIMOTO, Y.; LETTENMAIER, D. Satellite-based near-real-time estimation of irrigated crop water consumption. Journal of Geophysical Research, v. 114, n. D5, p. 1-14, 2009.

TOLEDO, C. E.; ARAÚJO, J. C.; ALMEIDA, C. L. The use of remote-sensing techniques to monitor dense reservoir newtorks in the Brazilian semiarid region. International Journal of Remote Sensing, v. 35, n. 10, p. 3683-3699, 2014. http://dx.doi.org/10.1080/014 31161.2014.915593.

\section{Contribuição dos autores}

Bruno Collischonn: Concepção do trabalho, processamento e análise dos dados, discussão dos resultados e redação do artigo.

Robin Thomas Clarke: Definição das metodologias e discussão dos resultados. 